\title{
La accesibilidad lingüística en el ámbito sanitario público de Andalucía. Posibilidades de intermediación en el contexto hispano-alemán
}

\author{
Cristina Martínez Fraile \\ Universidad de Sevilla
}

http:// dx.doi.org/ 10.12795/mAG Azin.2015.i23.04

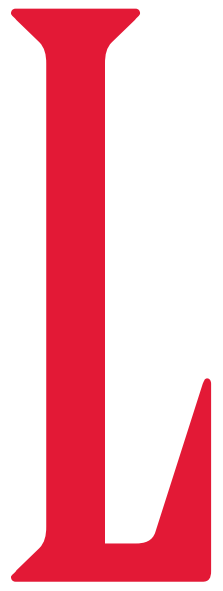

a asistencia sanitaria a pacientes extranjeros en Andalucía es un derecho reconocido más allá de los propios enunciados autonómicos porque se contempla no sólo como principio constitucional $^{1}$, sino que se eleva a un derecho europeo reconocido en la Carta de la Reforma de la Atención Sanitaria de Ljubljana $(1996)^{2}$. Andalucía también ha reaccionado, ante la creciente movilidad de ciudadanos europeos y no europeos, poniendo en marcha una serie de recursos humanos, como los puestos de intérpretes presenciales en los hospitales denominados Ventanillas Europeas de Información Sanitaria, y tecnológicos, como los sistemas de teletraducción de Salud Responde; asimismo, ha incorporado nuevos softwares de traducción médica, dirigidos a satisfacer las necesidades de una sociedad plural y plurilingüe. Ofrecer una asistencia adecuada a cada paciente comienza por asegurar la accesibilidad lingüística, esto es, por avalar el correcto proceso comunicativo entre el paciente y el personal sanitario. Pero una plena garantía de derechos se convierte en una utopía cuando se trata de requerir la presencia física, in situ y en el momento preciso, de intermediarios con la competencia lingüística y cultural adecuada, capaces de favorecer una situación de empatía $y$ de entendimiento pleno entre el personal sanitario y el enfermo (cfr. Yuste, J. 2010). Determinados testimonios reales advierten de que el ciudadano extranjero desconoce cuáles son sus derechos y que, en ocasiones, estas garantías prestacionales de atención sanitaria al ciudadano extranjero son sólo teóricas. Aunque no llegó a elevarse como queja formal, la experiencia vivida por una ciudadana alemana ${ }^{3}$, sin conocimientos de españoly escasos de inglés, en urgencias de un hospital público andaluz, sirvió de desencadenante en este estudio para analizar en profundidad qué derechos tiene el extranjero a la asistencia sanitaria pública y de qué recursos humanos y/o tecnológicos puede valerse para acceder a todas las prestaciones sanitarias en las distintas circunstancias, sobrevenidas y/o programadas. Como objeto de estudio se investigarán los medios disponibles por el Servicio Andaluz de Salud (SAS) así como las posibles formas de actuación de atención sanitaria al extranjero en los puestos de urgencias, en las consultas ordinarias de atención primaria y cómo se gestionan situaciones administrativas. Tomar como referente al paciente alemán servirá para dar a conocer dichas garantías prestacionales y para evitar posibles negligencias derivadas de problemas de accesibilidad lingüística.

\section{Legislación e información en el Portal del Servicio Andaluz de Salud}

En el mismo portal de la Junta de Andalucía se recoge la legislación que regula el derecho a la asistencia sanitaria en el ámbito de la región:

La Ley de Salud de Andalucía, en su artículo3, contempla que tienen derecho a las prestaciones y servicios de salud, tanto individual como colectivamente, los siguientes: Los españoles y los extranjeros residentes en cualquiera de los municipios de Andalucía. Los españoles y extranjeros no residentes en Andalucía que tengan establecida su residencia en el territorio nacional, con el alcance determinado por la legislación vigente. Los nacionales de Estados miembros de la Unión Europea tienen los derechos que resulten de la 


\section{Resumen:}

La cobertura sanitaria para el ciudadano extranjero es un derecho garantizado por la Unión Europea y esta comienza por contar con una adecuada información en su lengua materna. Analizando el sistema de garantía social sanitaria de Andalucía se advierte que aunque la Junta dispone de medios que favorecen la accesibilidad lingüística del ciudadano extranjero (como el servicio de teletraducción de Salud Responde o los Paneles de Comunicación) existen carencias que quedan por cubrir. Centrado en el ciudadano de lengua alemana se pretende dar a conocer las diferentes posibilidades de acceso a la sanidad pública de Andalucía, los sistemas de intermediación lingüística disponibles así como la detección de aspectos mejorables para hacer de nuestro sistema sanitario un modelo completamente accesible.

Palabras clave: cobertura sanitaria, sistema de garantía social sanitaria, accesibilidad lingüística, ciudadano extranjero, servicio de teletraducción.

\section{Zusammenfassung:}

Krankenversicherung für ausländische Bürger ist ein von der Europäischen Union garantiertes Recht. Eine richtige Information in seiner Muttersprache zu bekommen ist eine Mindestanforderung. Anhand der Analyse des Gesundheitssystems in Andalusien wird deutlich, dass zwar verschiedene Mittel (als Salud Responde, und die Paneles de Comunicación) zu Diensten der ausländischen Bürger bereitgestellt werden, es aber immer noch Mängel gibt. Am Beispiel deutscher Patienten sollen Möglichkeiten zur Verbesserung der sprachlichen Kommunikation zwischen spanischem Gesundheitssystem und ausländischen Patienten gezeigt werden, um die Gesundheitsversorgung in Andalusien für alle zugänglich zu machen.

Schlüsselwörter: Krankenversicherung, Gesundheitssystem, ausländische Bürger, Gesundheitsversorgung, Teletranslation. aplicación del derecho comunitario europeo y de los tratados y convenios, que se suscriban por el Estado Español y les sean de aplicación.[... $]^{4}$

El acceso a dichas prestaciones se realiza a través de la Tarjeta Sanitaria de Andalucía o de la Tarjeta Sanitaria Europea. La mayoría de los treinta derechos recogidos en la Carta de Derechos y Deberes expresan, de manera explícita o implícita, el concepto de la "accesibilidad lingüística", en tanto que se debe informar al usuario de manera adecuada sobre distintos aspectos de la sanidad que éste demande, como por ejemplo:

1. Recibir atención sanitaria en condiciones de igualdad, sin que pueda ser objeto de discriminación por razón alguna, respetando su personalidad, dignidad humana e intimidad.

2. Que se le ofrezca la atención, las prestaciones y servicios sanitarios disponibles que se consideren necesarios para cuidar su salud.

3. Recibir información en lenguaje comprensible usted, sus familiares o allegados de todo lo relacionado con su proceso, incluyendo diagnóstico, tratamiento, pronóstico, tiempo previsible de estancia en caso de ingreso y alternativas de tratamiento ${ }^{5}$.
En este sentido se entiende que posibilitar la comunicación entre el paciente extranjero y el médico o personal sanitario es un criterio de calidad con el que la sanidad pública tiene que cumplir, debiendo proporcionar los medios que la hagan factible.

El Portal de $\mathrm{SAS}^{6}$ debería ser el primer punto de encuentro, la carta de presentación para el ciudadano extranjero que va a viajar a la región y quiere informarse de las prestaciones sanitarias en caso de necesidad. Pero si un ciudadano de lengua alemana, de forma intuitiva, accede a la página del Servicio Andaluz de Salud para obtener alguna información se encuentra con la primera barrera lingüística, pues el portal únicamente presenta la versión española, sin posibilidad de acceder a la información a través ningún otro idioma que no sea el inglés: "Information on health care requirements for UK residents during their temporary stay in Andalusia. Campaña de colaboración Servicio Andaluz de Salud Consulado Británico”. A esta información se puede llegar a través de un enlace de la Tarjeta Sanitaria Europea ${ }^{7}$. Esto significa que el paciente de lengua alemana se ve obligado a comunicarse en inglés, idioma convertido en lingua franca, para poder acceder a la información. De hecho sí se informa en inglés acerca del servicio de teletraducción disponible en 64 idiomas: "Tele-translation service is also integrated in Health Responds and offers translations in 64 languages for those non-Spanish speakers who seek healthcare in the 
Andalusian Public Healthcare System. All Health Responds services are available too". Al margen de esta información, que al usuario alemán no le aparece de forma directa en la página de inicio, no hay disponible ninguna otra referencia aclaratoria en lengua extranjera en la página oficial.

\section{Prestaciones y recursos}

Aunque cualquier ciudadano extranjero puede verse involucrado en una situación como la que a continuación se describe, se va a tomar como ejemplo el supuesto de un paciente de lengua alemana con escasos o nulos conocimientos de español que quiere acceder a las prestaciones sanitarias de cualquier centro u hospital en Andalucía.

La interpretación presencial en las Ventanillas Europeas de Información Sanitaria (VEIS) y los Paneles de Comunicación como material auxiliar.

La atención sanitaria se entiende como un proceso personal y su calidad deriva, en última instancia, del grado presencial de intermediación humana entre el paciente y el personal sanitario (cfr. Yuste, J. 2010). En 1984, el INSALUD, en su Plan de Humanización a la Asistencia Sanitaria, apostaba por un sistema sanitario "lo más humano posible" y añadía que "la propia tecnificación de la medicina y la masificación despersonalizada, añade suficientes componentes para que el paciente se sienta frecuentemente desvalido, frente a esa situación que no domina [...]" (Gutierrez. 2012). Bajo esta óptica se entiende que la intermediación humana y presencial siempre es más deseable que cualquier intervención técnica por muy al alcance que esté de todos. La iniciativa de las llamadas Ventanillas Europeas de Información Sanitaria fue una muestra de la interpretación e intermediación presencial, donde al paciente extranjero se le ofrecía atención sanitaria en su mismo idioma. Este proyecto, iniciado en el Hospital de Carlos Haya de Málaga, consistió en la colocación de unos puntos de información con un servicio de interpretación en cinco idiomas (inglés, árabe, chelja, alemán y francés) ${ }^{9}$ que dejó de funcionar en el año 2012 por cuestiones administrativas quedando todo reducido al servicio de teletraducción Salud Responde. Esta acción fue muy criticada desde los sindicatos que elogiaban la labor de estos traductores presenciales: "hay veces en que la traducción por teléfono no es posible, como cuando el enfermo está en un quirófano sin anestesia general." ${ }^{10}$ En las Ventanillas Europeas la figura del intérprete, además de desempeñar labores de traducción, se tenía como mediador intercultural. En ocasiones su tarea iba más allá de la traducción lingüística, abordando cuestiones de convivencia, resolución de conflictos, etc. El mediador intercultural en el ámbito sanitario ha de contar con la competencia social que le obliga a ser conocedor de valores morales y religiosos, de tradiciones y de costumbres relacionadas también con el cuerpo y con las prácticas sanitarias. Asimismo, junto a esta competencia, se estima de gran importancia la capacidad para empatizar, formar u orientar (Navaza, B. / Estévez, L. / Serrano, J. 2009: 142). Dado que los recursos económicos sacrifican la garantía presencial y permanente de un personal cualificado en la interpretación, los mismos profesionales de la sanidad, situados en los puestos de urgencias, y llevados por la sentencia "de la necesidad nace el ingenio", han ideado otras alternativas de traducción para resolver problemas de comunicación lingüística. En formato electrónico ${ }^{11}$ o en

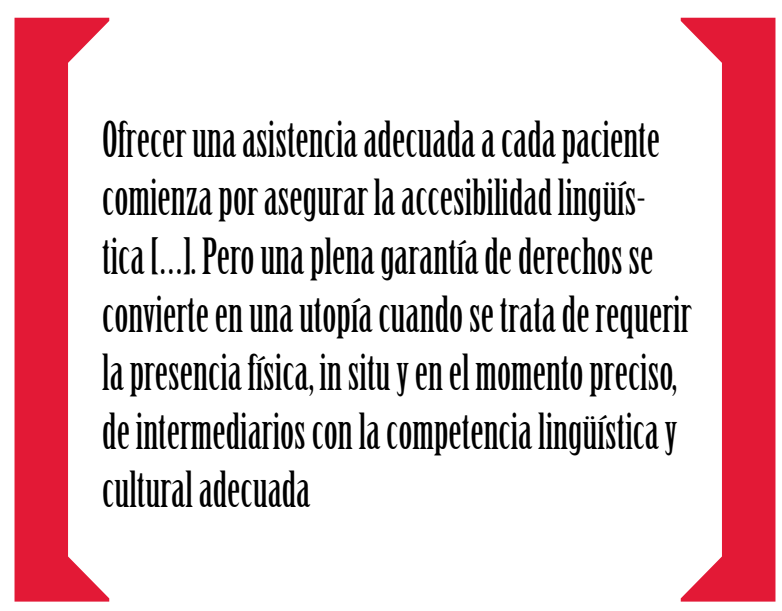

papel idearon un sistema pictográfico que, en forma de tabla, con emoticonos o dibujos, números y palabras, permiten al paciente extranjero informar del grado de dolor que tiene o de la dolencia en una determinada parte del cuerpo. De este modo, el paciente logrará expresar cómo se siente, qué necesita y obtendrá una mejor atención profesional adecuada a su padecer, una vez que el personal sanitario ha llegado a entenderlo.

Cabe citar la propuesta de un equipo de sanitarios de la UCI del Hospital "Punta Europa" de Algeciras ${ }^{12}$ y la de los profesionales de enfermería del Hospital "Virgen de las Nieves" de Granada que diseñaron dibujos con textos breves para comunicarse con los enfermos. El "Hospital Virgen Macarena" de Sevilla también ha creado un registro traducido en ocho idiomas, entre los que se encuentra la lengua alemana, para facilitar la comunicación en el área de Triage. ${ }^{13}$ El registro recoge un corpus de síntomas, variabilidad de su duración y antecedentes personales. En la elaboración de los paneles han colaborado todo un grupo de profesionales formado por enfermeras, terapeutas, 
logopedas, fisioterapeutas y traductores. $^{14}$ El Servicio Andaluz de Salud se ha valido de estos distintos modelos citados para crear los llamados Paneles de Comunicación y los ha incorporado a su sistema de atención sanitaria al extranjero traduciéndolos al árabe, al rumano, al ruso, al alemán ${ }^{15}$ y al francés. Aunque patentada fuera de la región andaluza, también es digna de mencionar otra innovación más sofisticada como la aplicación Pictorrino (Orduña, A. / Vicente, J. / Plaza, G., 2009), diseñada por ayudantes técnicos sanitarios de Fuenlabrada y comercializada como herramienta de comunicación con pacientes incapacitados para hablar y con usuarios extranjeros. Pictorrino aún no cuenta con la lengua alemana entre sus cinco idiomas (español, inglés, francés, chino y árabe).

\section{La atención telefónica a través de Salud Responde}

La garantía prestacional de los derechos fundamentales deviene dependiente de los recursos económicos y humanos disponibles; precisamente la imposibilidad física y económica de disponer permanentemente de un equipo de intérpretes presenciales en cada centro hospitalario se convierte en un límite de dichas garantías (cfr. Böckenförde, 1993) y esto ha motivado la adopción de otras medidas como la teletraducción o la instrucción del personal sanitario en el manejo de nuevos softwares y aplicaciones móviles dirigidos a paliar las carencias que pueden derivarse de una interpretación no presencial. En el año 2003 la Consejería de Salud introduce un sistema de información y gestión sanitaria a través de la atención telefónica, Salud Responde. Entre su amplia cartera de servicios se recoge el "Servicio de Traducción Lingüística”, cuya labor gestiona Dualia como empresa proveedora y responsable. A través del planteamiento de un caso real se puede valorar el grado de accesibilidad lingüística de este servicio y para ello se plantea el supuesto en el que un ciudadano de lengua alemana llama al servicio de Salud Responde 902505060 o al número 061 de urgencias. En la primera toma de contacto se advierte la principal barrera lingüística, pues el enfermo o familiar del enfermo se halla ante un teleoperador que directamente lo atiende en español. Si el usuario de lengua alemana no sabe identificarse, el audaz operador deberá deducir el idioma para poder activar el servicio de teletraducción. Superada esta primera barrera, la alternativa de la teletraducción consiste en un procedimiento de interpretación telefónica en el que se establece un "triálogo" entre el mediador/intérprete, el paciente y el personal sanitario. En una consulta médica o en un servicio de urgencias será el personal sanitario el que haga uso de este servicio de teletraducción ante la recepción de un ciudadano extranjero al que no entiende. El sanitario realizará una llamada para la que se vale de un terminal móvil y un biauricular, un sistema que permite la "conversación a tres". Así se describe en la página del Servicio Andaluz de Salud:

A través de una simple llamada de teléfono, el profesional contacta con el call center desde donde se facilita la conexión inmediata con el intérprete del idioma requerido, quien realiza una traducción simultánea de la conversación entre el ciudadano que no habla español y el profesional. ${ }^{16}$

La conversación se traduce en un tiempo real. Con un servicio de 46 idiomas, de los que 11 están disponibles durante 24 horas todos los días del año (entre los que se encuentra el alemán) y el resto sólo de 8.00 a 18.00 en días laborables, este servicio de teletraducción permite dar respuesta a cualquier consulta general sobre medicina, urgencias y trámites administrativos. ${ }^{17}$ La teletraducción se presenta como la mejor opción después de la interpretación presencial, si se atiende a diferentes criterios. Entre ellos se considera la disponibilidad del teletraductor, no sujeta ni al momento ni al lugar donde se requiera su atención. Evitar el desplazamiento físico del intérprete ahorra no sólo tiempo en condiciones urgentes sino que también supone una reducción en los costes que, en comparación, requiere la interpretación presencial. Asimismo, el abanico de lenguas disponibles es muy superior al que pueda ofrecer un equipo de traductores presentes en un centro; la confidencialidad e imparcialidad del teletraductor también garantizan el derecho a la intimidad del paciente (cfr. Navaza, B. / Estévez, L. / Serrano, J, 2009: 143). Si bien este sistema de la teletraducción garantiza la accesibilidad a las distintas prestaciones sanitarias por parte del ciudadano extranjero también presenta ciertas carencias. Por ejemplo, esta comunicación a tres puede resultar complicada e incómoda para los pacientes en determinadas situaciones como en un parto o un postoperatorio donde el enfermo, en una situación de dolor, tenga que estar sujeto o dependiente de un teléfono para poder ser comprendido. Es cuando más se requiere la presencia física de un mediador/intérprete. Asimismo, se pueden sumar problemas como los técnicos (acústica, conexión y otras interferencias y ruidos). El mismo intérprete telefónico debe tener en cuenta que pueden presentársele situaciones sobrevenidas para las que necesita estar en alerta permanente y guardar la calma. Junto a la teletraducción existen en el mercado algunos softwares multilingües o aplicaciones móviles interactivas 
como Universal Doctor Speaker, ${ }^{18}$ que no dejan de ser útiles pero tampoco dejan de ser simples herramientas de apoyo para el sanitario o el paciente porque la comunicación aparece limitada por un corpus cerrado de preguntas y respuestas. Este hecho obliga a depender, en última instancia, de un intérprete

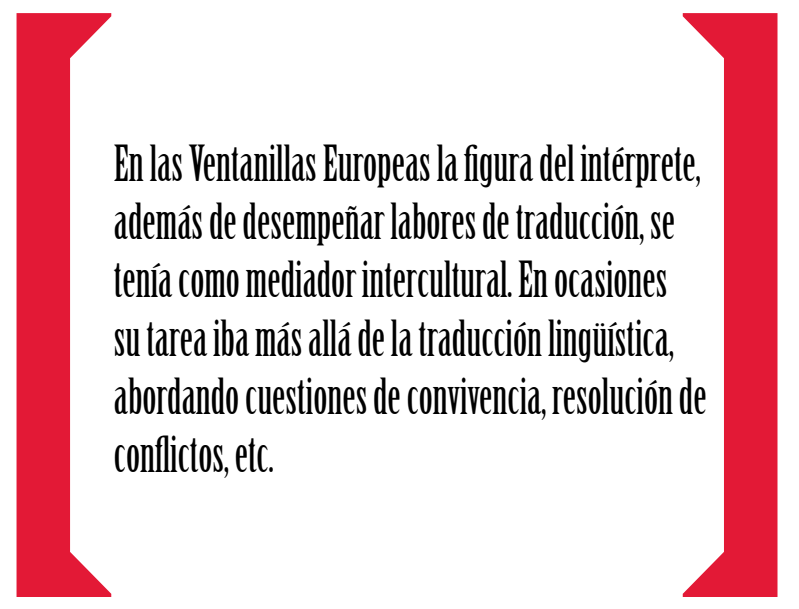

que, de forma presencial o no, acabará captando esa información no verbal y/o no sistematizada por el programa informático. Donde sí resulta útil esta aplicación es para temas de gestión administrativa, tales como dar citas, prescripciones, para otra información de carácter técnico o como misma herramienta auxiliar de un intérprete.

\section{La figura del mediador o intérprete. Su perfil y su situación laboral}

El mediador-intérprete sanitario es una figura poco definida laboralmente si se atiende a criterios como su formación especializada, su tipo de contrato, sus honorarios o su flexibilidad horaria y/o disponibilidad permanente. En España, a diferencia de Canadá, Australia, Estados Unidos y Reino Unido, ${ }^{19}$ para esta figura de intérprete en servicios públicos no existen acreditaciones oficiales aunque sí másteres profesionalizantes tales como el Máster Universitario en Comunicación Intercultural, Interpretación y Traducción en los Servicios Públicos de la Universidad de Alcalá o el Máster Universitario de Investigación en Traducción e Interpretación de la Universidad Jaume I en el que se incluyen asignaturas como Metodología de la investigación en traducción médica y Comunicación Intercultural mediada en el ámbito sanitario. Otro máster que puede cursarse en la Universidad Jaume I es el Máster Universitario en Traducción MédicoSanitaria con las asignaturas de Mediación intercultural en el ámbito sanitario y Técnicas de interpretación en los servicios públicos sanitarios. Según Corsellis (2008: 59), los candidatos deben reunir cualidades como el conocimiento del servicio público y de su funcionamiento así como de su código ético. Igualmente, desde el punto de vista de las habilidades traductológicas, han de presentar fluidez escrita y oral en los pares de lenguas para transferir de forma exacta los significados. En un nivel más de concreción Kelly (2008: 75), en su estudio Telephone interpreting. A comprehensive guide to the profession, anade las cualidades del traductor telefónico, entre las que cita la experiencia en interpretación consecutiva con habilidades como la memoria y la capacidad para anotar y organizar una comunicación que, a priori puede presentarse enrevesada a causa de una emergencia o de la falta de claridad del interlocutor. También se ha de valorar la experiencia profesional en otros contextos telefónicos, por ejemplo en puestos como "atención al cliente" donde técnicas sobre cómo la vocalización, la respiración y las pausas, entre otros aspectos, son factores que deben tenerse en cuenta. Asimismo, debe saber superar problemas que pudieran distorsionar la conversación, desde los técnicos hasta los meramente lingüísticos (acentos, tonos, ritmo, etc.). ${ }^{20}$

En el ámbito sanitario público de Andalucía, desde la desaparición de los puestos de interpretación presencial de las llamadas Ventanillas Europeas en el Hospital Carlos Haya de Málaga, sólo se puede indagar en la figura del teletraductor que presta sus servicios en la empresa Dualia, como empresa proveedora de la Consejería de Salud y Bienestar de la Junta de Andalucía. Según la información a la que se puede acceder en la página de la Junta, el profesional traductor está cualificado para resolver problemas relacionados con trámites administrativos, urgencias, cartera de servicios, etc. porque cuenta con una formación sobre el sistema sanitario así como de atención a pacientes y familiares. Pero para conocer con más detalles el perfil de los teletraductores contratados por Dualia se instó a la empresa a ofrecer detalles sobre la formación de los mismos. El "teleoperador-traductor/intérprete" contratado tiene que acreditar la titulación de intérprete o traductor; en caso de no contar con una titulación universitaria el personal de recursos humanos valorará otros méritos, como el bilingüismo, que garanticen la capacidad para desarrollar dicha tarea; también se tendrán en cuenta la especialización a través de másteres o cursos de formación. Esta empresa no facilitaba datos acerca de los honorarios que recibían sus trabajadores. Sobre la remuneración por tareas de interpretación en hospitales únicamente se conoce que los siete intérpretes voluntarios, sin relación contractual, que formaban parte del cuerpo de las VEIS cobraban 600 euros 
en forma de gratificación. Ya se adelantaba al principio que este grupo de interlocutores no cuenta con el merecido reconocimiento profesional, reconocimiento que se traduce en precarios contratos de trabajo aunque se le requiere una formación especializada para poder desempeñar plenamente su tarea. M.I. Abril en su estudio de 2006 La interpretación en los Servicios Públicos: caracterización como género, contextualización y modelos de formación. Hacia unas bases para el diseño curricular apunta que existen carencias en la formación reglada o cursos de especialización en este ámbito y que a veces existen contrataciones de intérpretes no cualificados. El intérprete del ámbito sanitario no debe tener necesariamente una formación en medicina aunque, por cuestiones sumamente delicadas como las vitales, es imprescindible que disponga de una formación experta en la materia y que domine adecuadamente el tecnolecto de los dos pares de lenguas en este campo.

En primer lugar, éste ha de enfrentarse a un vocabulario extraordinariamente amplio, pues se calcula que existen unos 210.000 términos médicos, aunque el léxico que un profesional utiliza, de hecho, se puede reducir, sin embargo, a unos 6000 a 8000 términos. De la cantidad global, unos 80.000
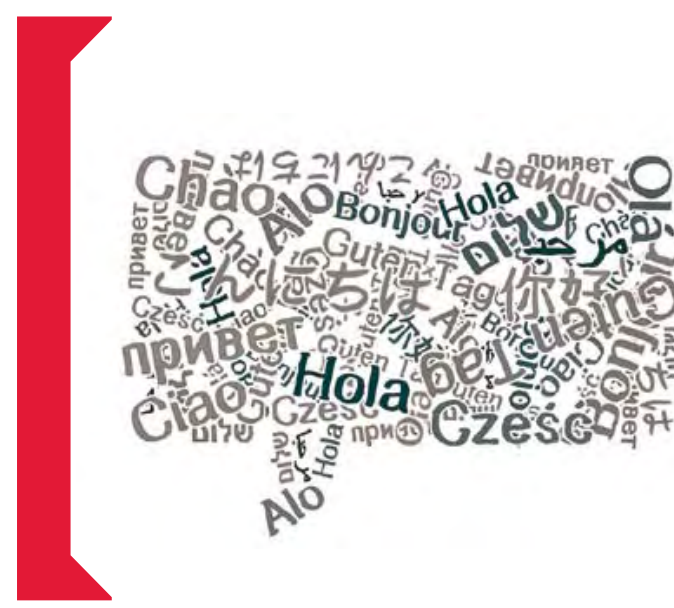

detectar sus carencias. A través del supuesto de un paciente de lengua alemana que quiere acceder a la sanidad pública en Andalucía se ha conseguido dibujar el mapa de atención sanitaria al ciudadano extranjero. Andalucía también se ha sumado a las políticas europeas sanitarias incorporando recursos para ofrecer de manera adecuada sus prestaciones a ciudadanos extranjeros de habla no española. Aunque sólo la interpretación presencial garantizaría plenamente la accesibilidad lingüística, y por ende la adecuada atención sanitaria, ésta se vuelve utópica cuando las limitadas partidas económicas obligan a hacer uso de otros procedimientos. Se devalúa, de esta forma, la figura del intérprete/mediador en el ámbito sanitario, que no es tarea fácil porque requiere contar con habilidades y competencias lingüísticas, culturales y socio-comunicativas, muchas de las cuales se adquieren a través de una formación académica experta mientras que otras se nutren corresponden a nombres de medicamentos, otros 10.000 suman las diversas de partes del cuerpo, órganos y partes de los diferentes órganos; se estima en 60.000 los términos que hacen referencia a las distintas funciones de los órganos y, por último, existen unos 60.000 nombres de enfermedades, afecciones, exploraciones y operaciones. Este vocabulario es prácticamente inabarcable, incluso para los médicos mismos. A la gran cantidad de ramas y especialidades que componen las ciencias biosanitarias ha de sumarse además una innovación casi constante de términos con el avance de la ciencia (Parra 2006: 37).

\section{Conclusiones}

A veces situaciones que producen desconcierto sirven para analizar cómo funcionan determinados sistemas de garantía social, para conocer sus prestaciones así como para de la experiencia. Existen aún numerosas lagunas en el campo de la formación académica especializada en este ámbito de mediadores/intérpretes biosanitarios en Andalucía (en España existen algunos másteres profesionalizantes), donde la oferta es muy reducida. También, a diferencia de otros países, cabría reclamar la creación de agencias o entidades que acreditasen, de una forma homogénea, la capacitación del profesional de este sector, un factor que no sólo le conferiría a la profesión calidad sino también fiabilidad. Este gran esfuerzo del mediador/intérprete no cuenta con su merecido reconocimiento como profesional, traduciéndose en contratos precarios, pese a llegar a ser la pieza clave que resuelve los problemas de accesibilidad lingüística en la atención sanitaria. Su presencia se ha ido sustituyendo por puestos de teletraducción, que se presentan como una opción altamente competitiva y válida para resolver problemas de comunicación para con pacientes extranjeros, logrando reducir el gasto económico pero, a veces, en aras de sacrificar ciertos aspectos propios de la comunicación no verbal que subyacen en una interacción presencial. El paciente alemán se encuentra dentro del grupo de habla no hispana que cuenta con esta cobertura lingüística de teletraducción permanente durante 24 horas todos los días del año. En determinadas circunstancias, bien por la falta de 
gravedad o por evitar la intervención de un intermediario, los mismos profesionales sanitarios, valiéndose además de sus habilidades sociales, especialistas e interpretativas, disponen de recursos como los Paneles de Comunicación de base iconográfica y traducidos en distintos idiomas entre los que se encuentra el alemán; con ellos consiguen solventar de una forma más diligente algunos casos que sólo requieren primeros auxilios.
Con esta reflexión también quiere abrirse las puertas a otras investigaciones centradas en el turista o emigrante español. En este panorama actual de emigración de todo tipo de personas con o sin formación en idiomas, sería interesante estudiar qué barreras lingüísticas encuentra el ciudadano español en el extranjero para acceder a las prestaciones médicas y de qué recursos se vale el país receptor para resolverlas.
1 Ley Orgánica 4/2000, en los términos previstos en el artículo 12 del 11 de enero, sobre los derechos y libertades de los extranjeros en España y su integración social.

2 https://www.aecc.es/SobreElCancer/bibliotecadedocumentos/ Documents/Declaraciones\%20internacionales/carta\%20europea\%20 de\%20los\%20derechos\%20de\%20los\%20pacientes.pdf (consultado el 26 de marzo de 2016)

3 Se respeta su deseo de quedar en el anonimato.

4 http://www.juntadeandalucia.es/salud/sites/csalud/contenidos/ Informacion_General/c_2_c_4_prestaciones_sanitarias/quien_tiene_ derecho_y_como_se_accede (consultado el 26 de marzo de 2016).

5 http://www.juntadeandalucia.es/salud/sites/csalud/contenidos/ Informacion_General/c_2_c_1_carta_de_derechos_y_deberes/ Derechos (consultado el 26 de marzo de 2016).

6 http://www.juntadeandalucia.es/salud/sites/csalud/contenidos/ Informacion_General/c_2_c_1_carta_de_derechos_y_deberes/ Derechos (consultado el 26 de marzo de 2016).

7 http://www.juntadeandalucia.es/servicioandaluzdesalud/principal/ documentosAcc.asp?pagina=gr tseu ropea (consultado el 26 de marzo de 2016)

8 lbid.

9 http://www.juntadeandalucia.es/servicioandaluzdesalud/principal/ noticia. asp?codcontenido=4031 (consultado el 26 de marzo de 2016).

10 http://www.diariosur.es/v/20130112/malaga/gerencia-carlos-hayaprescinde-20130112.html (consultado el 26 de marzo de 2016).

11 Véase el ejemplo de la aplicación Pictorrino (en la cita n.13)

12 http://www.juntadeandalucia.es/servicioandaluzdesalud/principal/ documentosAcc.asp?pagina=pr_desa_Innovacion11 (consultado el 26 de marzo de 2016).
13 Zona de recepción de los enfermos en urgencias donde se realiza la primera diagnosis una vez que el paciente describe sus síntomas.

14 Cfr. Plan Integral de Cuidados de Andalucía en http:// www.juntadeandalucia.es/servicioandaluz desalud/principal/ documentosAcc.asp?pagina=gr_estratcuidados (consultado el 26 de marzo de 2016).

15 http://www.juntadeandalucia.es/servicioandaluzdesalud/library/ plantillas/externa.asp?pag=/contenidos/../contenidos/gestioncalidad/ DICE/Paneles/PanelAleman.pdf (consultado el 26 de marzo de 2016).

$16 \mathrm{http}: / / w w w . j u n t a d e a n d a l u c i a . e s / s e r v i c i o a n d a l u z d e s a l u d /$ principal/noticia.asp?codcontenido=4031 (consultado el 26 de marzo de 2016).

17 http://www.juntadeandalucia.es/servicioandaluzdesalud/ principal/documentosacc.asp?pagina=gr_teletradui_b3 (consultado el 26 de marzo de 2016).

18 Universal Projects and Tools S.L. crea en 2011 este software multilingüe que también puede encontrarse en versión de aplicación móvil. Se presenta como traductor multilingüe de los idiomas español, alemán, bokmål noruego, catalán, chino simplificado, francés, inglés, italiano, japonés, polaco, portugués, rumano, ruso, somalí, árabe para facilitar la comunicación "entre los pacientes o los usuarios de la sanidad y cualquier trabajador sanitario, se encuentren donde se encuentren y provengan de donde provengan". Véase la descripción al completo. [Documento disponible en Internet en https://itunes.apple. com/es/app/universal-doctor-speaker-traductor/id389202856? $\mathrm{mt}=8$.] (consultado el 26 de marzo de 2016).

19 Estos países cuentan con un sistema de acreditación nacional oficial. Cfr. Ibid. Navaza, B., Estévez, L., y Serrano, J. (2009: 143).

20 Resulta de gran interés la entrevista de un trabajador de Dualia. [Documento disponible en Internet en http://www.traducirco. com/2013/12/como-funciona-la-interpretacion.html.] (consultado el 26 de marzo de 2016). 
Bibliographie

Abril, M. I. (2006), La interpretación en los Servicios Públicos: Caracterización como género, contextualización y modelos de formación. Hacia unas bases para el diseño curricular. Tesis doctoral, Universidad de Granada, Granada.

Böckenförde, A.-W. (1993), Escritos sobre derechos fundamentales, Nomos Verlag, Baden-Baden.

Corsellis, A. (2008), Public Service Interpreting. The First Steps. Basingstoke: Palgrave McMillan.

Gutierrez, R. (2011/2012), (DES) HUMANIZACIÓN en (de) la atención sanitaria [Documento disponible en Internet en http://www. regimen-sanitatis.com/2011/12/ deshumanizacion-en-de-la-atencion. html] (consultado el 26 de marzo de 2016).

Kelly, N. (2008), Telephone interpreting. A comprehensive guide to the profession. Trafford Publishing, Victoria.

Navaza, B., Estévez, L. y Serrano, J. (2009), "Saque la lengua por favor". Panorama actual de la interpretación sanitaria en España, Panace@ 10 (30), [Revista digital disponible en Internet en http://www.medtrad.org/ panacea/IndiceGeneral/n30_tribunaNavazaEstevezSerrano.pdf].

Orduña, A., Vicente, J. y Plaza,

G. (2009) Comunicación de necesidades en el paciente laringectomizado: Pictorrino en Acta Otorrinolaringológica Española, $60(05)$ [Documento disponible en Internet en http://www.elsevier.es/ es-revista-acta-otorrinolaringologicaespanola-102-articulo-comunicacionnecesidades-el-pacientelaringectomizado-13141555].

Parra, P. (2006), "Módulo sobre el léxico biosanitario", en Máster Universitario en Traducción de textos con fines específicos, Interpretación y Doblaje de las lenguas española y alemana, Universidad de Sevilla, Sevilla.

Parrilla, L. (2014), El reto de una interpretación comunitaria de calidad: buceo en las necesidades formativas y análisis crítico de un corpus de interacción oral en el contexto biosanitario y de servicios sociales español. Universidad de Málaga, Servicio de Publicaciones, Málaga.

Yuste, J. (2010) Intérpretes de papel para mujeres embarazadas inmigrantes [Documento de Internet disponible en http://joseyustefrias.com/index.php/ blog/item/interpretes-de-papel-paramujeres-embarazadas-inmigrantes. $\mathrm{html}]$

Otros documentos web (consultados el 26 de marzo de 2016):

Carta de la Reforma de la Atención Sanitaria de Ljubljana (1996) [Documento de Internet disponible en https://www.aecc.es/SobreElCancer/ bibliotecadedocumentos/Documents/ Declaraciones\%20internacionales/ carta $\% 20$ europea $\% 20 \mathrm{de} \% 201$ os $\% 20$ derechos $\% 20 \mathrm{de} \% 2010 \mathrm{l} \% 20$ pacientes.pdf].

Entrevista a un trabajador de la teletraducción [Documento de Internet disponible en http://www.traducirco. com/2013/12/como-funciona-lainterpretacion.html]. Información sobre la herramienta informática Universal Doctor Speaker. [Documento disponible en Internet en https://itunes.apple.com/es/app/ universal-doctor-speaker-traductor/ $\mathrm{id} 389202856 ? \mathrm{mt}=8$ ].

Junta de Andalucía: derecho a las prestaciones sanitarias [Documento de Internet disponible en: http://www. juntadeandalucia.es/salud/sites/csalud/ contenidos/Informacion_General/ c_2_c_4_prestaciones_sanitarias/quien tiene_derecho_y_como_se_accede]. Junta de Andalucía: Tarjeta Sanitaria Europea [Documento de Internet disponible en http://www.juntadeandalucia. es/servicioandaluzdesalud/principal/ documentosAcc.asp?pagina $=$ gr tseuropea].

Junta de Andalucía: servicio de teletraducción [Documeto de Internet disponible en http://www.juntadeandalucia. es/servicioandaluzdesalud/principal/ documentosacc.asp?pagina=gr_teletradui b3].
Junta de Andalucía: noticia sobre el servicio de las Ventanillas Europeas de Información Sanitaria [Documento de Internet disponible en http://www.juntadeandalucia.es/ servicioandaluzdesalud/principal/noticia. asp? codcontenido=4031].

Junta de Andalucía: panel de comunicación. Versión alemana. [Documento de Internet disponible en http://www.juntadeandalucia. es/servicioandaluzdesalud/library/ plantillas/ externa.asp?pag=/contenidos/../ contenidos/gestioncalidad/DICE/Paneles/ PanelAleman.pdf].

Junta de Andalucía: creación de recursos para eliminar las barreras lingüísticas [Documento de Internet disponible en http http://www.juntadeandalucia. es/servicioandaluzdesalud/principal/ documentosAcc.asp?pagina=pr_desa Innovacion1].

Junta de Andalucía: Carta de Derechos de la Ciudadanía [Documento de Internet disponible en:http://www. juntadeandalucia.es/salud/sites/csalud/ contenidos/Informacion_General/ c 2 c 1 carta de derechos y deberes/ Derechos].

Noticia sobre la eliminación de las Ventanillas Europeas de Información Sanitaria [Documento de Internet disponible en http://www.diariosur. es/v/20130112/malaga/gerencia-carloshaya-prescinde-20130112.html].

Plan Integral de Cuidados de Andalucía [Documento de Internet disponible en http:/www.juntadeandalucia.es/ servicioandaluzdesalud/principal/ documentosAcc.asp?pagina $=$ gr estratcuidados]. 